\title{
Pruebas de evaluación Saber y PISA en la Educación Obligatoria de Colombia
}

\section{Assessment in Compulsory Education in Colombia: The Saber and PISA tests}

\author{
Luz Amparo Sanabria James*1 \\ sanabriajames@gmail.com \\ M. Carmen Pérez Almagro** \\ maricarmen.perez@aulagrupo.es \\ Luis Enrique Riascos Hinestroza*** \\ riascosh@hotmail.com \\ * Coordinadora Nodo Caribe Insular de REDLENGUAJE, San Andrés, Colombia \\ ** Universidad Americana de Europa, UNADE, México \\ *** Rector, Institución Educativa Monteloro, Tuluá del Valle, Colombia
}

\begin{abstract}
Resumen:
INTRODUCCIÓN. Esta investigación se centra en la revisión documental a las políticas de evaluación externas a los estudiantes de educación obligatoria en Colombia. Se trata de las Pruebas Saber y la prueba internacional PISA. MÉTODO. Este trabajo describe cómo se evalúa en el país y cuáles han sido sus resultados en los niveles de $3^{\circ}$, $5^{\circ}$ y $9^{\circ}$ de educación básica, y, $11^{\circ}$ de educación media obligatoria. Para comenzar, se presenta el contexto con las características de estas dos evaluaciones, así como las políticas y acciones abordadas para mejorar la dinámica y calidad de la oferta. RESULTADOS. Por último, se señalan las tendencias en los resultados del proceso educativo de un país que se debate en el dilema de la cobertura educativa, en el que, a pesar de los esfuerzos por mejorar la igualdad y
\end{abstract}

\begin{abstract}
:
INTRODUCTION. This article reviews the external evaluation policies of students in compulsory education in Colombia. Specific attention is paid to the "Saber" and the international PISA tests. METHOD. This work describes how evaluation is undertaken in the country and what its results are for the $3^{\text {rd }}, 5^{\text {th }}$ and $9^{\text {th }}$ levels of basic education and the $11^{\text {th }}$ level of compulsory secondary education. Firstly, the context and characteristics of these two evaluations are presented, as well as the policies and actions addressed to improve the dynamics and quality of the teaching offer. RESULTS. Results show that the country is immersed in a debate about educational coverage which, despite the efforts made to improve equality and quality, has not led to improvements in students' aca-
\end{abstract}

1 Dirección para correspondencia (correspondence address):

Luz Amparo Sanabria James. Universidad Nacional de Colombia, Sede Caribe, San Andrés Isla. Carretera Circunvalar San Luis Free Town 52 - 44, San Andrés, San Andrés y Providencia (Colombia). 
la calidad, no se logran las mejoras en los desempeños de los escolares. DISCUSIÓN. El trabajo histórico-documental aparece como representativo para conocer el grado competencial de los alumnos ya que supone un campo de actividad al que contribuye el conjunto de la comunidad educativa.

\section{Palabras claves:}

Evaluación educativa; pruebas estandarizadas; políticas de evaluación; Colombia; Pruebas Saber; ICFES; PISA. demic performances. DISCUSSION. Historical-documentary work seems to foster interprofessional relationships in that it involves the whole educational community.

\section{Key words:}

Educational assessment; standardized testing; assessment policies; Colombia; Pruebas Saber; ICFES; PISA.

\section{Résumé:}

INTRODUCTION. Cette recherche se concentre sur une analyse documentaire des politiques d'évaluation externe des élèves de l'enseignement obligatoire en Colombie. II s'agit des tests Pruebas de Saber et du test international PISA. MÉTHODE. Ce travail décrit comment on évalue les élèves dans le pays et quels ont été les résultats des $3^{\mathrm{e}}, 5^{\mathrm{e}}$ et $9^{\mathrm{e}}$ niveaux de lienseignement de base et du $11^{\mathrm{e}}$ niveau de lienseignement secondaire obligatoire. Pour commencer, le contexte est présenté avec les caractéristiques de ces deux évaluations, ainsi que les politiques et actions abordées pour améliorer la dynamique et la qualité de l`offre. RÉSULTATS. Enfin, on présente les tendances dans les résultats du processus éducatif dıun pays qui sont discutées dans le dilemme de la couverture éducative, et dans lequel, malgré les efforts pour améliorer liégalité et la qualité, les améliorations de performance des écoliers ne sont pas atteintes. DISCUSSION. Le travail historique et documentaire semble être représentatif pour reconnaître le niveau de compétence des élèves puisqu'il constitue un champ d`activité auquel contribue liensemble de la communauté éducative.

\section{Mots-clés:}

Évaluation éducative; tests normalisés; politiques dıévaluation; Colombie; Pruebas Saber; ICFES; PISA.

Fecha de recepción: 17-01-2019

Fecha de aceptación: 14-05-2020

\section{Introducción}

Para la UNESCO la educación además de ser un derecho fundamental es un proceso de transformación que permite saber si los estándares establecidos en este ámbito se adecúan a la realidad y demandas que la sociedad exige a los jóvenes. De ahí, la importancia de la evaluación de los estudiantes para tomar decisiones convenientes y ajustar los planes de mejora a las políticas locales y nacionales. Ahora bien, al analizar esa evaluación es posible entender las dinámicas, los procesos y sus características y determinar los alcances en los distintos niveles de la educación en general. 
Este análisis de las pruebas de evaluación externas a los estudiantes de primaria y secundaria en Colombia se ha realizado teniendo en cuenta las políticas que diseña y reglamenta el Estado como son las Pruebas Saber del Instituto Colombiano para el Fomento de la Educación Superior (ICFES), y, el programa internacional para la evaluación de estudiantes (Programme for International Student Assessment, PISA). El valor numérico de estos diagnósticos permite identificar los logros, fortalezas y debilidades de los estudiantes para evaluar y retroalimentar aspectos institucionales como la pertinencia del currículo y la efectividad de la enseñanza (Braun y Kanjee, 2006; Ayala-García, 2015). Por ese motivo, este trabajo ha considerado describir las dinámicas sobre evaluación en la educación obligatoria de Colombia para comparar los resultados en la educación básica y media, con el fin de establecer las tendencias en los resultados del proceso. Para ello, se ha llevado a cabo una revisión bibliográfico-documental desde un modelo prospectivo general que permite elaborar un análisis estadístico de la situación.

La metodología se ha desarrollado tomando como referente el concepto de revisión documental que se inicia con la recopilación, revisión, análisis, selección y extracción de información de diversas fuentes (Hurtado de Barreda, 2000). Revisados los conceptos de educación, calidad y evaluación desde las distintas normativas que se han tenido en cuenta en la oferta educativa y las propuestas de integrar los aspectos curriculares, los recursos y disponiblidad de las instalaciones físicas a las reflexiones de mejora en el sector, se ha procedido a revisar los resultados de las pruebas externas que han presentado los estudiantes en los distintos niveles del sistema educativo en Colombia. Esto ha permitido establecer las tendencias en los resultados.

Desde la investigación descriptiva documental se puede analizar los procesos para mostrar las dinámicas en las políticas de evaluación y en las competencias evaluadas para determinar la tendencia y el alcance en la gestión educativa del quinquenio 2014 a 2018, así como de los próximos años.

\section{Antecedentes y marco teórico-conceptual}

En América Latina, las políticas educativas se han enmarcado desde dos ideologías que inciden en la calidad de la evaluación, la formación y 
la reformulación de las políticas (Reimers, 2003, p. 11). La primera se encuentra propulsada por el Estado, con las prácticas institucionales y la cultura de la comunidad educativa local. Mientras que la segunda está establecida por las élites que están a cargo de las políticas públicas, centrándose de esta forma sólo en decisiones externas a los procesos educativos del aula y las realidades de los contextos. Es así que Horn, Wolff \& Vélez (1992) señalan que la información de la evaluación puede ser de provecho para el sistema cuando se acompaña de un programa para el incremento de la calidad, porque la medición del aprendizaje escolar, en sí misma, no produce un aumento en el rendimiento. De allí que, para establecer las metas cuantitativas, es necesaria la evaluación de las estrategias, de las combinaciones de los insumos, de las técnicas de producción, así como de la asignación de recursos y de esfuerzos para lograr esas metas (Horn et al., 1992, pp. 5-9) y no sólo de decisiones externas a las dinámicas institucionales. Ahora bien, por un lado tampoco se puede descartar que el proceso que involucra la aplicación de una prueba asigna un valor numérico a los resultados para identificar los logros de los estudiantes, sus fortalezas y debilidades; y por otro, brinda así una retroalimentación al evaluar aspectos institucionales como la pertinencia del currículo y la efectividad de la enseñanza (Braun y Kanjee, 2006; UNESCO, 2000 en Ayala-García).

Es importante entender como presenta Castells (2000) que en este siglo XXI no se asiste únicamente a una globalización económica, sino también de la ciencia, la tecnología y la información. Este fenómeno está presente tanto en los medios de comunicación masiva y multimedia, como en las nuevas formas de comunicación a través de Internet. Es por ello, que se podría pensar en la posibilidad de que las evaluaciones reflejasen estas capacidades y habilidades de los estudiantes. De esta manera la educación tendría que ser objeto de conocimiento científico, que, aplicando un método riguroso en el diseño, la implementación y su desarrollo llevaría a resultados de metas relevantes para el progreso del ser humano (Pérez, 2000, pp. 263-265).

Desde estas reflexiones se hace un alto en el contexto colombiano, donde la educación se define como un proceso de formación permanente, personal, cultural, social y fundamentado en la concepción integral de la persona, su dignidad, sus derechos y sus deberes. Lo que permitiría pensar en las propuestas de Horn et al. (1992), Castells (2000) y Pérez (2000) como viables en esta definición y podría ser gradual según su es- 
tructura educativa pues contempla una educación inicial, la de preescolar; continuada en la básica primaria con cinco grados, seguida de una básica secundaria de cuatro grados para desembocar en la educación obligatoria media que son los dos últimos grados que permite al joven obtener el título de bachiller. Esta acreditación le concede la oportunidad de seguir a la educación superior (Programa Ser Pilo Paga).

Con respecto a esto, el país se ha debatido entre el dilema de la cobertura y la calidad, situación que le ha llevado a evaluar la dinámica de acceso al sistema para mejorar la cobertura. Por lo que se podría pensar que se diluyeron las acciones en torno a la evaluación de las estrategias, las combinaciones de los insumos, las técnicas de producción y la asignación de recursos para atender a la evaluación interna y externa en las que participan los estudiantes de cada uno de los niveles ofertados en el país.

En cuanto a los resultados en pruebas externas estandarizadas, AyalaGarcía (2015) muestra que el esfuerzo que ha realizado el Ministerio por mejorar la alta desigualdad entre los niveles educativos lo lleva a centrarse efectivamente en el trabajo de mejorar la cobertura. Sin embargo, esta acción no se evidencia en resultados de los desempeños escolares. Los casos se pueden ir señalando a lo largo de estos años de análisis. Un ejemplo se observa en las competencias matemáticas de la prueba nacional Saber/2013 donde un 44\% de los estudiantes de Colombia quedó en el nivel bajo. A nivel internacional, en esta misma competencia se ubicó en el puesto 62 entre los 65 países que participaron en la prueba PISA 2012 (Ayala-García, 2015, pp. 1-2). A cierre de este periodo de análisis la dinámica según el ICFES (2018) registraba que para el año 2017 la tendencia creciente que los resultados habían presentado en 2016 se detuvo en $3^{\circ}, 5^{\circ}$ y $9^{\circ}$ grado tanto en matemáticas como en lenguaje.

Con el fin de determinar la calidad de la educación y definir políticas de mejora, los estudiantes se enfrentan a ambos diagnósticos, con criterios unificados y estandarizados. Es de observar que el Decreto 1290 emanado por el Ministerio de Educación Nacional en 2009, faculta a las instituciones educativas a determinar su Sistema de Evaluación interna haciendo que la forma de evaluar en el aula a los educandos sea particular, propia y autónoma en cada institución y dependa del modelo educativo y pedagógico de su Proyecto Educativo Institucional (PEI) establecido en la Ley 115 o Ley General de Educación del Congreso de la República de Colombia (1994). Por lo que es pertinente contemplar 
en las dos evaluaciones estas habilidades de los medios que se reflejan en las capacidades y habilidades de los estudiantes para dar respuesta a las competencias científicas y matemáticas, además de la comunicativa, buscando un punto de equilibrio entre estas.

\section{La evaluación en la educación: sus implicaciones}

En el foro de la Organización de Estados Iberoamericanos (OEI) sobre evaluación de la educación, Metas para 2021, se evidencia que la evaluación se ha convertido en un instrumento indispensable para pilotar el desarrollo de los sistemas educativos y valorar el impacto de las políticas de educación (OEI, Conferencia Iberoamericana de Ministros de Educación, 2009). De esta forma se debe atender la oferta en educación, revisar las acciones directivas, administrativas y académicas propuestas para entender los resultados que da la evaluación y orientar políticas para la mejora de la calidad (VV.AA., 2010, p. 120). El análisis al acceso, progresión y culminación de primaria, realizado por la OEI durante 2007 y 2008, muestra que no se ha cumplido con la meta en la cadena de formación (figura 1). Se requiere que todos los estudiantes estén en el sistema, reflejando un progreso real y efectivo de habilidades y conocimientos para un desarrollo social (VV. AA., 2010). Situación en la que el nivel de rezago y retención de Colombia (figura 2) muestra desventaja según la UNESCO (VV.AA., 2010).

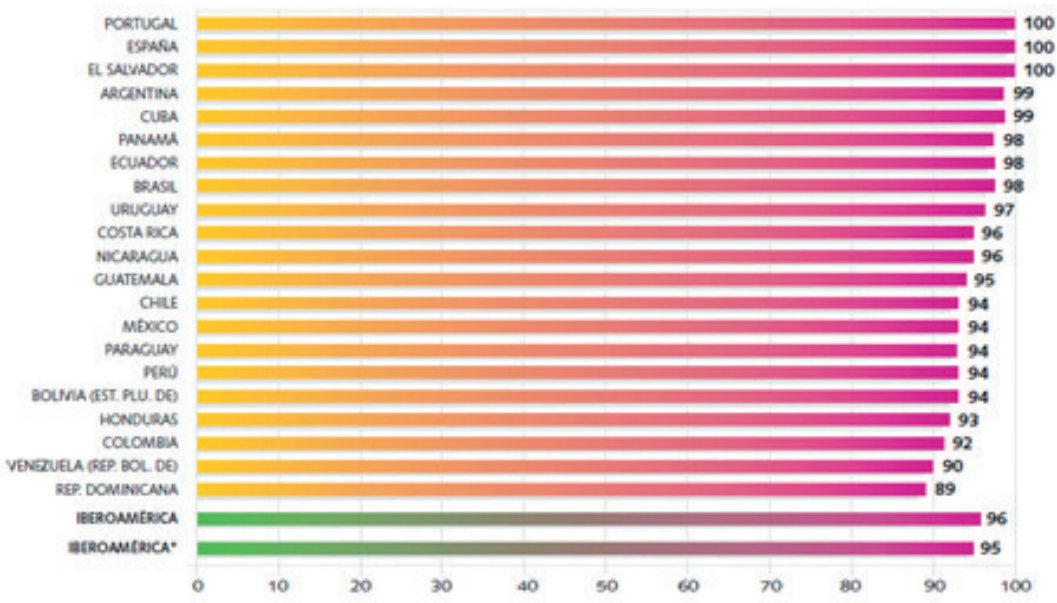

Figura 1. Iberoamérica, tasa de matrícula neta en enseñanza primaria 2007-2008 (VV.AA., 2010, p. 38). 


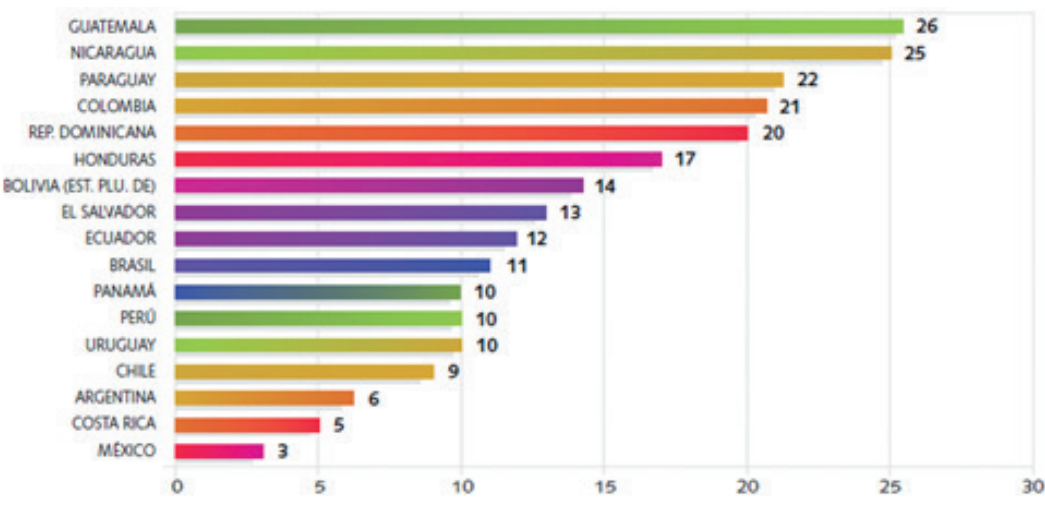

Figura 2. Atraso escolar de los niños entre 9 y 11 años (VV.AA., 2010, p. 39).

La situación en Colombia permite observar que, a medida que se avanza en los niveles educativos, hay debilidad en acceso y progresión oportuna en cada uno de los niveles. En la figura 1 se observa que un $92 \%$ de la población es atendida, sin embargo, un $21 \%$ presentan atraso en el sistema (figura 2). El sistema logra ubicar un $71 \%$ de los jóvenes en el nivel de secundaria (figura 3); con un escaso $40 \%$ en el nivel de secundaria superior (figura 4), ciclo que se orienta a la especialización, con demanda de docentes para atender estos perfiles, OEI, Organización de Estados Iberoamericanos para la Educación, la Ciencia y la Cultura (2010). De esta forma Colombia se ubica entre los últimos seis países de la región que completan el ciclo de educación obligatoria de sus jóvenes. Situación que llama a la reflexión para atender esta realidad de manera concreta. No sólo con facilidades al acceso sino a la retención, con propuestas innovadoras en el aula, uso de distintas herramientas didácticas y tecnológicas para resolver situaciones del contexto estudiantil. Complementado con locaciones físicas adecuadas, oportunidades de integrarse a distintos grupos de emprendimiento e investigación creando o fortaleciendo redes académicas de docentes y de estudiantes en los distintos niveles. 


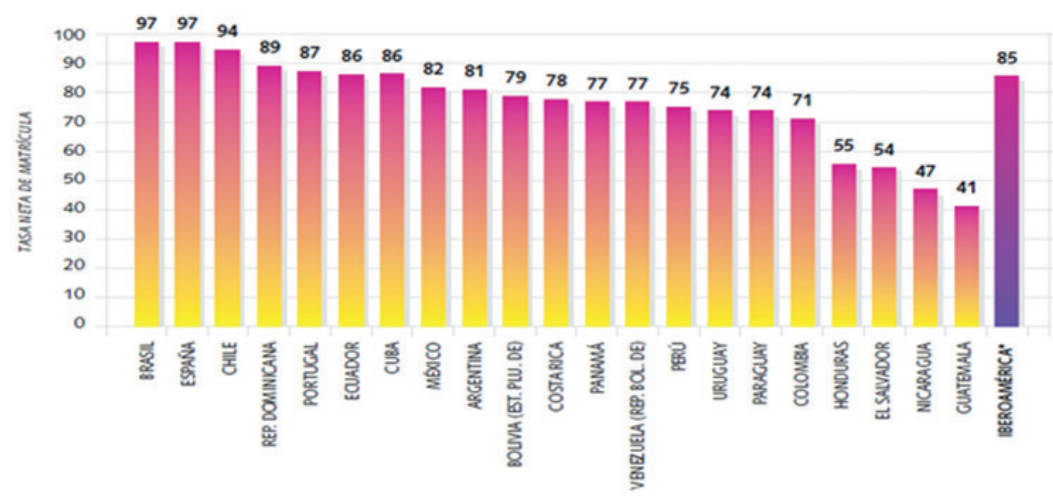

Figura 3. Tasa de matrícula neta en enseñanza secundaria 2008 (VV.AA., 2010, p. 43).

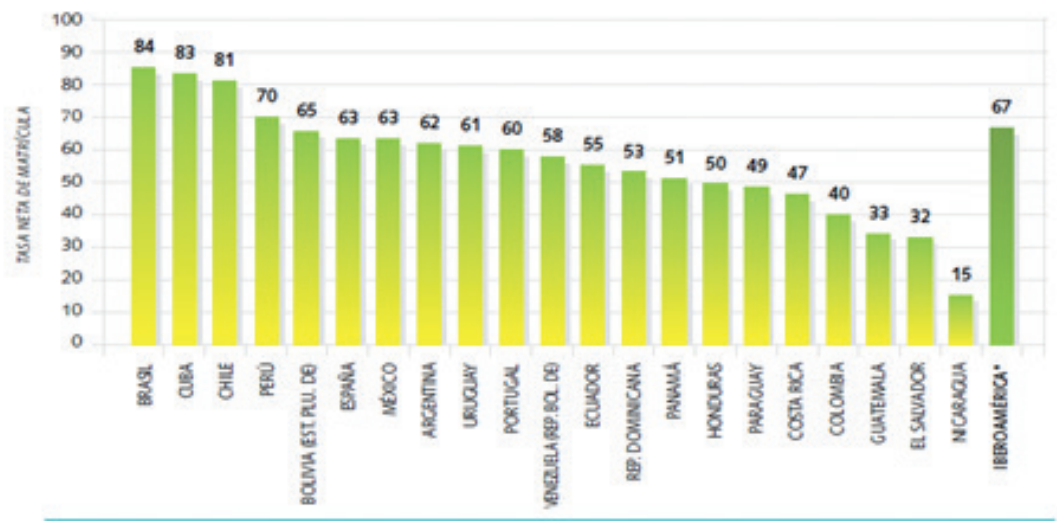

Figura 4. Tasa de matrícula neta en enseñanza secundaria superior 2008 (VV.AA., 2010, p. 46).

La evaluación como proceso continuo y permanente tiene diferentes tipos de implicaciones dependiendo el para qué y con quién se realiza, del contexto y sus condiciones de vulnerabilidad, y, las dinámicas sociales y educativas en que esta se realice. Así como las características que determina cada área o disciplina al referirse al cómo y con qué evaluar. Lleva a pensar que esta depende entonces de factores externos e internos y que las instituciones deben revisar, analizar, pormenorizar y priorizar estos aspectos físicos, de recursos y estrategias, así como de las responsabilidades a cada uno de los agentes del sector y sus roles (estudiante, padres, docentes, directivos, administrativos). 


\section{La evaluación a los estudiantes}

Si como se mencionó anteriormente, al hablar de evaluación es necesario retomar el contexto en el que se realiza y las razones por las cuáles se hace. El contexto que convoca este análisis es el de la educación y para ello las políticas se enmarcan según The People's Movement for Human Rights Education (2005) en la "Declaración Universal de los Derechos Humanos, los Pactos Internacionales, la Convención de los Derechos del Niño y otros tratados y declaraciones internacionales" (p. 1) que facilita a todos los humanos hacer uso del derecho a la educación.

Las razones se enmarcan en la necesidad de un servicio de calidad. De acuerdo con la Oficina Regional de Educación de la UNESCO para América Latina y el Caribe, la calidad se "concibe como un medio para que el ser humano se desarrolle plenamente como tal, ya que gracias a ella crece y se fortalece como persona y como especie que contribuye al desarrollo de la sociedad, transmitiendo y compartiendo sus valores y cultura" OREALC/UNESCO, Organización de las Naciones Unidas para la Educación, la Ciencia y la Cultura (2008, p. 6).

Un segundo concepto de calidad hace referencia a la operacionalización de la calidad o nivel de logros en Educación. Aquí se emplea el término calidad como sinónimo de efectividad y eficiencia que permiten formulación de políticas para su mejoramiento, haciendo oportuna la evaluación en todos los niveles, Arancibia (1997, p. 5) De esta forma, las reflexiones en torno a la evaluación de la calidad educativa en América Latina y el Caribe realizada en Santiago de Chile, registra que la evaluación del rendimiento de los estudiantes es con el fin de determinar el grado de eficacia de los sistemas y mejorar la calidad. Pero también para demostrar en qué medida se logran los objetivos educativos y el aprendizaje en cada nivel UNESCO (VV.AA., 2008, p. 6).

\section{La evaluación en el contexto normativo de Colombia}

En Colombia el proceso de evaluación escolar ha pasado por una serie de normas que caracterizan los distintos intentos de mejorar resultados. Se puede señalar el Decreto Ley 088 del 22 de enero de 1976 que reestructuró el Sistema Educativo en materia de evaluación, estableció en su artículo 8, la promoción automática en básica primaria; reglamentado 
por el Decreto 1469 del 3 de agosto de 1987. Normas que permitieron la permanencia de los niños y niñas en el Sistema, pero no ayudaron con la calidad de los resultados (figuras 5 a 7 ).

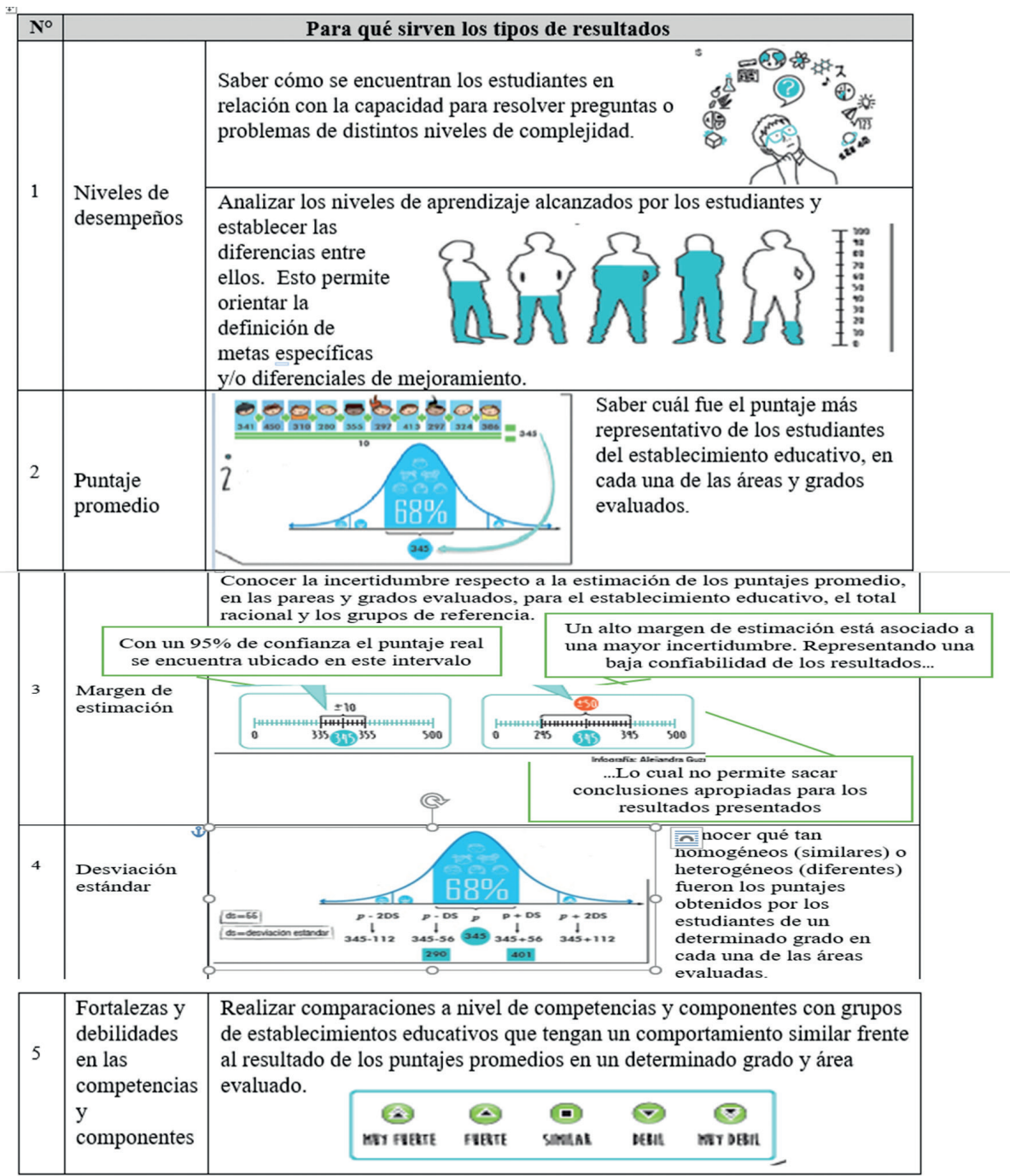

Figura 5. Consideraciones para el análisis de resultados en Pruebas Saber $3^{\circ}, 5^{\circ}$ y $9^{\circ}$ en Colombia.

Elaboración propia a partir de ICFES, 2015, p. 15. 


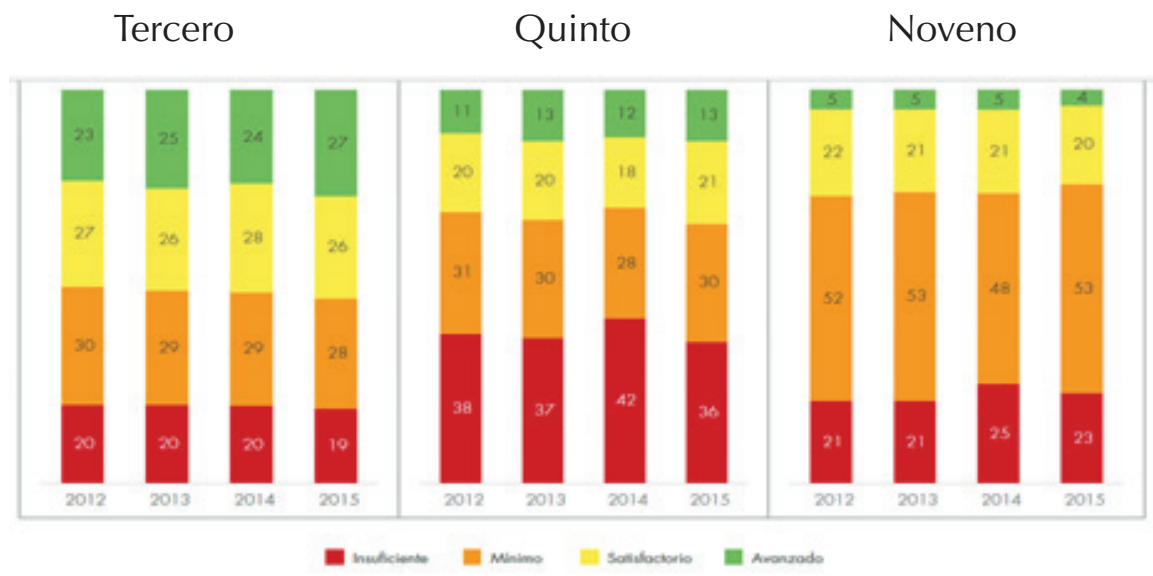

Figura 6. Desempeños en matemáticas - Pruebas Saber $3^{\circ}, 5^{\circ}$ y $9^{\circ}$ entre 2012 2015 (ICFES, 2018, p. 3).

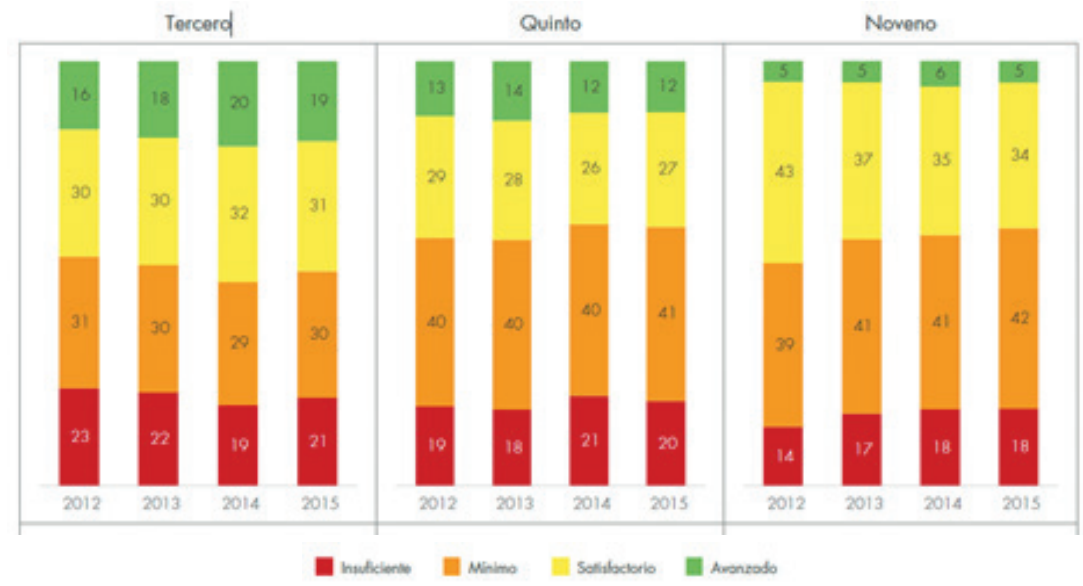

Figura 7. Desempeños en lenguaje - Pruebas Saber $3^{\circ}, 5^{\circ}$ y $9^{\circ}$ entre 2012-2015 (ICFES, 2018, p. 3).

Para atender lo curricular en todos los niveles de la oferta, surge el Decreto 1419 del 17 de julio de 1978. En el artículo 3 establecía que el proceso educativo debe estar centrado en el estudiante, su desarrollo armónico e integral y los programas deben tener un equilibrio entre lo teórico y lo práctico para promover el estudio y comprensión de los problemas nacionales e internacionales. El Decreto 1002 del 24 de abril de 1984 estableció el plan de estudios, áreas, modalidades, organización y distribución de los tiempos, metodologías y evaluación para todos los niveles obligatorios del sistema, precisándose que la evaluación no era 
para la asignación de notas sino para mejorar el proceso enseñanza y la calidad del aprendizaje (art. 19). Para hacer efectiva la promoción o paso de un grado a otro, la Resolución 17486 de 1984 permitió expresar los resultados de la evaluación en forma descriptiva para dar paso a la evaluación cualitativa.

La Ley 115 del 8 de febrero de 1994 planteó la educación como un proceso de formación permanente, personal, cultural y social, en la concepción integral de la persona, su dignidad, derechos y deberes. El Decreto 1860 de 1994 estableció que la evaluación sería continua, integral y cualitativa expresada en informes descriptivos, favoreciendo los conocimientos, valores y actitudes, el desarrollo de capacidades y habilidades. De esta manera el docente dispondría de una información para reorientar o consolidar sus prácticas pedagógicas, identificar características y ritmos de aprendizajes personales, ofreciéndole al estudiante oportunidades para aprender.

El Decreto 230 del 11 de febrero de 2002, reglamentación espacial de la ley 115 de 1994, dictó normas en materia de currículo, evaluación, promoción de los educandos y evaluación institucional. En su artículo 1 definió la evaluación como "...el proceso permanente mediante el cual es valorado el nivel de desempeño de los estudiantes en relación con las competencias básicas". El artículo 2 expresaba la escala de valoración nacional excelente, sobresaliente, aprobado, no aprobado, presentado los descriptores que indicarían las debilidades y fortalezas de los educandos. Se definieron criterios, procesos, procedimientos para la evaluación y promoción anticipada de grado y las nivelaciones, garantizando la promoción del 95\% de los educandos. El Decreto 230 es derogado por el Decreto 1290 de 2009 que define los ámbitos en los que se realizará la evaluación del aprendizaje y los clasifica en internacional, nacional e institucional. Se establece la autonomía de las instituciones educativas para poner en marcha su propio sistema de evaluación de los aprendizajes de acuerdo al PEI y ajustándose a una escala nacional que se referencia en término de niveles de desempeño.

En la búsqueda de resultados en pruebas nacionales e internacionales, el sector ha realizado múltiples intentos ajustados a las distintas normas señaladas, pero sin transformar significativamente los resultados, pues los esfuerzos se concentran en adiestrar a los estudiantes en la tipología de las evaluaciones, en los contenidos de las asignaturas o áreas y en el manejo de tipos de textos. Se sigue haciendo un manejo fragmentado de 
la información, poca presencia de la transversalización e interdisciplinariedad de la información como respuesta a situaciones reales, lo que dificulta al estudiante dar respuesta a posibles alternativas de solución que se le plantean en las evaluaciones. Es de señalar también que se siguen observando problemas de adecuación de los espacios, ambientes escolares, formación de maestros, número de estudiantes por capacidad instalada en aulas, así como una reciente insistencia de que lo evaluado en pruebas externas nacional o internacional debe ser lo atendido de manera exclusiva en el aula. En este sentido, obtener buenos puntajes se ha convertido en una prioridad para los directivos y en motivo de competencia entre instituciones -animado por los ránquines de algunos medios, que sirve de norte a padres para seleccionar el colegio de sus hijos Rodríguez (2018). Aquí se puede fortalecer la propuesta del aula si se realiza un diagnóstico de las necesidades reales y sus alternativas de solución que en un trabajo integral docente- estudiante-escuela- comunidad permitiría los resultados esperados.

\section{La evaluación como estrategia}

Mejorar la cantidad y la calidad de la educación es uno de los desafíos que enfrenta Colombia en su propósito de acelerar el crecimiento económico y ofrecer mayores posibilidades de bienestar a la población (Delgado, 2014, p. 123). De allí que una de las estrategias ha sido la evaluación a los estudiantes en todos los niveles de la cadena de formación.

En Colombia los estudiantes de la educación básica participan en las pruebas internas, Pruebas Saber, que en 1991 comenzó con aplicaciones muestrales a estudiantes de $5^{\circ}$ y $9^{\circ}$ y que durante el 2002 y 2003 se realizaron las primeras pruebas censales en las áreas de lenguaje, matemáticas, ciencias naturales, competencias ciudadanas y a partir de 2005 se incluyó el área de ciencias sociales (MEN, 2006, p.1). El Ministerio y el ICFES publican los resultados que luego analizan con las secretarías de educación sugiriendo estrategias a desarrollar. Las secretarías de educación a su vez, realizan talleres con las instituciones, con el fin de elaborar los planes de mejoramientos según necesidades manifiestan en los desempeños de los escolares. En la figura 5 se observa un esquema orientador que busca optimizar estos planes a partir de los datos arrojados en las pruebas anuales. Los directivos y sus docentes se reunen 
durante las semanas de desarrollo institucional, al finalizar el primer y segundo semestre del año escolar, y utilizando las cartillas con los resultados de su institución, fortalecen los planes de mejoramiento y consolidan los planes de áreas. Se busca así mejorar los resultados en pruebas.

\section{Las pruebas externas para estudiantes}

Los estudiantes del último año de la educación escolar calendario A y B, participan en las Pruebas Saber $11^{\circ}$, conocidas anteriormente como pruebas ICFES, dependencia del Servicio Nacional de Pruebas (SNP) que surgió a partir de la reestructuración realizada al Servicio de Admisión Universitaria y Orientación Profesional creado en 1966 e inició su aplicación a los bachilleres del país en agosto de 1968 (Grupo de Investigación sobre Pruebas Masivas en Colombia, 2008). En 1980 se reglamenta el examen convirtiéndose en requisito para el ingreso a cualquier programa de pregrado en el territorio nacional (Colombia Aprende. La red del conocimiento, 2016, p.1).

En el primer examen se evaluó aptitud matemática, aptitud verbal, razonamiento abstracto, relaciones espaciales, ciencias sociales y filosofía, química, física, biología e inglés y se presentaron transformaciones en 1980, 2000 y 2005. Desde 2007 la estructura presenta dos grandes componentes, uno común y otro flexible. Las pruebas del núcleo común son en lenguaje, matemática, física, química, biología, ciencias sociales, filosofía e inglés; en el componente flexible, cada estudiante puede optar una profundización entre: lenguaje, matemáticas, biología o ciencias sociales. La prueba interdisciplinar permite escoger entre medio ambiente y violencia y sociedad (Colombia Aprende-La red del conocimiento, 2016, p.2). A partir de los resultados individuales e institucionales, la institución puede detectar las debilidades y fortalezas para plantear los planes de mejoramiento de las áreas y las políticas en el Plan de Mejoramiento Institucional (PMI).

A los estudiantes de la educación básica y media del país se les aplican pruebas de modelos internacionales cuyos resultados permiten a las autoridades educativas analizar el impacto de las acciones desarrolladas en el sector. Es así que Colombia además de la prueba PISA formó parte de otros análisis: Trends in International Mathematics and Science Study (TIMSS) en 1995; en el Primer Estudio Internacional Comparativo del 
LLECE (1997); en el Estudio Internacional de Cívica, de la IEA (1999); y en el Progress in International Reading Literacy Study (PIRLS), en 2001 (Las distintas pruebas, 2006, p.1).

Desde el año 2000 y cada 3 años, los estudiantes de 15 años independientemente del grado en el que se encuentran, son convocados a las evaluaciones PISA, que evalúa conocimientos y habilidades relacionados con la comprensión lectora, matemática y científica. En 2005, Colombia aplicó una prueba piloto a 1.720 estudiantes de 55 instituciones escolares. Desde 2006 participa en estas pruebas, en esa oportunidad se presentaron 5.250 de 150 instituciones (Las distintas pruebas, 2006, p.1).

Los resultados de las pruebas PISA para los años 2006 y 2009 registran aumentos en las tres áreas (matemáticas, lectura y ciencias). No obstante, el país se ubica en los rangos inferiores entre los países participantes y por debajo de países latinoamericanos como Chile, México y Argentina (OECD, 2010) en Delgado 2014, p.123-124. Sobre la participación en 2015 el ICFES (2017) muestra que se incrementó la cobertura de participación con respecto a la aplicación de 2006, pasando de un $60 \%$ a un $75 \%$ disminuyendo en esta prueba la tasa de preguntas que quedaron sin respuesta con respecto a la anterior, $6 \%$ de las preguntas de lectura y matemáticas, y solo el 4,5\% de las preguntas de ciencias.

En cuanto a los resultados en 2018 la OECD (2019) muestra que los estudiantes de Colombia obtuvieron un rendimiento menor que la media de la OCDE en lectura (412 puntos), matemáticas (391) y ciencias (413).

\section{La Educación Básica y sus resultados}

Como se mencionó anteriormente, el esquema educativo de Colombia comprende los ciclos de básica primaria del grado primero a quinto y básica secundaria, de sexto a noveno. En estos niveles de la educación colombiana, los estudiantes han participado en la Pruebas Saber 3, 5 y 9, aplicadas por el ICFES tanto al sector oficial y privado como a los dos calendarios académicos A y B, que se puede observar en las disposiciones legales.

"Con la Ley 715 de 2001 se establece el carácter obligatorio y censal de esta evaluación y su realización cada tres años. A partir de 2002 son evaluados los estudiantes de los grados $5^{\circ}$ y $9^{\circ}$ de calendarios A y B de todos los esta- 
blecimientos oficiales y privados del país, y en 2012 se incluyó la evaluación de estudiantes de grado $3^{\circ}$. Como parte de la política de evaluación del MEN, desde 2012 se realizan también aplicaciones anuales de estas pruebas para los grados $3^{\circ}, 5^{\circ}$ y $9^{\circ}$. Para completar la estructura de aplicaciones de la educación básica, se está desarrollando desde 2015 la incorporación de pruebas para grado $7^{\circ}$. (...) se evalúan las áreas de Lenguaje y Matemáticas en los cuatro grados y de Competencias Ciudadanas y Ciencias Naturales en $5^{\circ}$, $7^{\circ}$ y $9^{\circ}$. El esquema de aplicaciones contempla que siempre se evalúen las áreas de lenguaje y matemáticas, mientras que la evaluación de las pruebas de competencias ciudadanas y ciencias naturales se realice alternadamente. (...) se produce resultados a nivel de los establecimientos educativos, sobre las competencias en cada una de las áreas y grados evaluados y se acompaña de información que se recolecta con los cuestionarios de factores asociados y de información sociodemográfica". (ICFES, Ministerio de Educación, 2015, parr. 4 a 6$)$

El ICFES publica cada año los resultados de las pruebas con información institucional así como el análisis de los mismos. Las tablas muestran el agregado nacional del porcentaje de estudiantes en cada uno de los cuatro niveles de desempeño para las áreas de lenguaje y matemáticas durante el periodo 2012 a 2015. Se observa que la mayor cantidad de estudiantes se acumula en el nivel de desempeño mínimo y en insuficiente los del grado $5^{\circ}$, situación que precisa trabajar en torno a estas competencias ýa que el número de estudiantes que siguen en niveles bajos es significativo a pesar que los estándares básicos de competencias del Ministerio de Educación Nacional establecen que todos los estudiantes del país estén, al menos, en el nivel de desempeño "satisfactorio" (ICFES, 2016, p. 2).

Al tomar como ejemplo una de las pruebas internacionales de lenguaje, matemáticas y ciencias aplicadas en el país, los resultados demuestran que las instituciones oficiales se encuentran por debajo de los promedios obtenidos por las instituciones privadas (tabla 1). Referentes que invitan a que los planes de mejoramientos no sólo atiendan las prácticas de evaluaciones tipo, como se frecuenta sino fortalecer toda la oferta escolar para ser pertinentes con las demandas que se le presentan a los estudiantes. El trabajo por proyectos de aula es una apuesta que facilita la comprensión de los contenidos, ayuda a la aplicación en el contexto para la propuesta de alternativas de solución debido a la transversaliza- 
ción e interdisciplinariedad de la información. De esta forma, se desarrollan las habilidades y destrezas en los estudiantes y se les facilita el análisis que deben hacer para dar respuesta en las pruebas externas.

Tabla 1

Puntajes promedios en Pruebas Pisa 2006, 2009 y 2012

\begin{tabular}{llllllllll}
\hline Tipo de & \multicolumn{3}{l}{ Matemáticas } & \multicolumn{3}{l}{ Lectura } & \multicolumn{3}{c}{ Ciencias } \\
\cline { 2 - 10 } colegio & $\mathbf{2 0 0 6}$ & $\mathbf{2 0 0 9}$ & $\mathbf{2 0 1 2}$ & $\mathbf{2 0 0 6}$ & $\mathbf{2 0 0 9}$ & $\mathbf{2 0 1 2}$ & $\mathbf{2 0 0 6}$ & $\mathbf{2 0 0 9}$ & $\mathbf{2 0 1 2}$ \\
\hline Público & 361 & 369 & 369 & 378 & 400 & 394 & 379 & 389 & 392 \\
\hline Privado & 415 & 430 & 441 & 425 & 468 & 476 & 429 & 455 & 455 \\
\hline Total & 370 & 381 & 376 & 385 & 413 & 403 & 388 & 402 & 399 \\
\hline
\end{tabular}

Fuente: Barrera, Maldonado y Rodríguez (2012).

\section{La situación en la Educación Media}

Este nivel comprende los grados diez y once de la educación obligatoria. El resultado de las pruebas Saber $11^{\circ}$ en Colombia es un tema de interés general para las instituciones educativas, las autoridades locales y nacional, que convoca a directivos, docentes y estudiantes a distintas actividades de preparación para mejorar resultados (tablas 2 y 3 ), puesto que la oferta de incentivos para los jóvenes y sus instituciones va en aumento como se lee en el comunicado de prensa emitido por el Ministerio de Educación y el ICFES.

En el camino para ser el país más educado de América Latina en 2025 hemos logrado un sacudón a la calidad de la educación, los resultados de esta Prueba Saber 11 muestran la respuesta de los estudiantes frente al programa de becas Ser Pilo Paga 2", afirmó la Ministra de Educación, Gina Parody. El incentivo de 11.000 créditos condonables disponibles para los puntajes ubicados en el $7 \%$ superior hizo que los potenciales pilos se trazaran la meta de obtener esos resultados, situación que estiró la distribución de los puntajes logrando que el corte subiera en 8 puntos frente al año 2014. Por lo tanto, el corte en la prueba Saber 11 para los potenciales beneficiarios del programa Ser Pilo Paga pasó de un año a otro de 310 a 318 (Los Tres Editores SAS, 2015). 
Tabla 2

Departamentos que presentan crecimiento porcentual de estudiantes en puestos del 1 al 400

\begin{tabular}{lll}
\hline Departamento & $\mathbf{2 0 1 0}$ & $\mathbf{2 0 1 4}$ \\
\hline Cundinamarca & $40 \%$ & $42 \%$ \\
\hline Boyacá & $38 \%$ & $39 \%$ \\
\hline Arauca & $35 \%$ & $36 \%$ \\
\hline San Andrés & $26 \%$ & $35 \%$ \\
\hline Meta & $34 \%$ & $35 \%$ \\
\hline Antioquia & $30 \%$ & $31 \%$ \\
\hline Guajira & $21 \%$ & $24 \%$ \\
\hline
\end{tabular}

Fuente: Ministerio de Educación de Colombia, 28 de noviembre de 2014.

En la figura 8 se observa las grandes diferencias que hay entre los resultados de los estudiantes ubicados entre los puestos 1 al 400 en los departamento según crecimiento porcentual de estudiantes. La dinámica muestra también los cambios efectuados en cada uno en los resultados de los años 2005, 2010 y 2014, que lleva a cada una de las Entidades Territoriales a los correspondientes análisis para evaluar las acciones educativas.

Al respecto la Ministra de Educación de Colombia, manifiesta:

El examen de Estado es aplicado en Colombia desde 1968 a los estudiantes (...) de grado 11, con el fin de conocer, (...) cómo se encuentra la calidad de la educación media en el país, además de ser requisito primordial para el ingreso de los jóvenes a la Educación Superior. Este año fueron evaluados en total 548.704 estudiantes de colegios oficiales y privados de Calendario A, que representan el $84 \%$ de la matrícula total en todo el país (Centro Virtual de Noticias de Educación, 2014, p.1). 


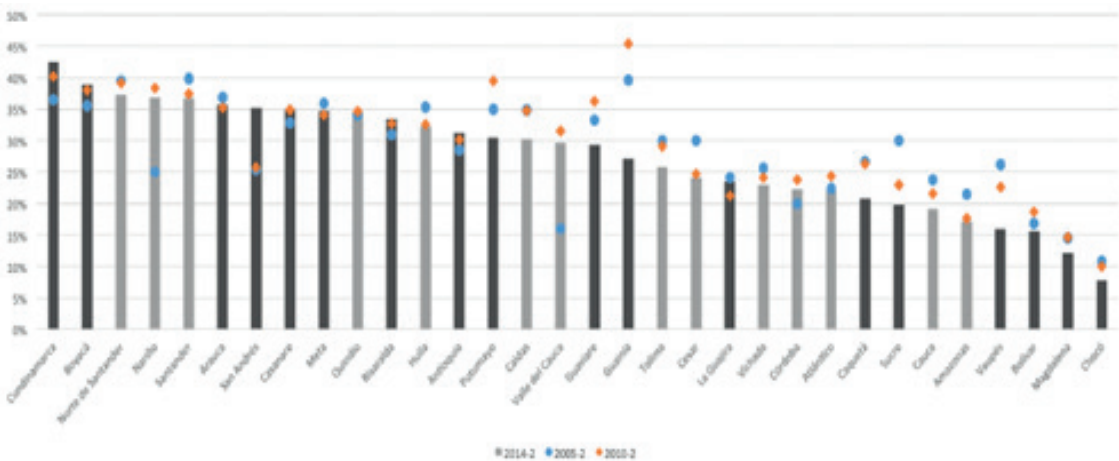

Figura 8. Colombia. Resultados de las Pruebas Saber $11^{\circ}$ en Entidades Territoriales Certificadas. (Ministerio de Educación de Colombia, 28 de noviembre de 2014).

En la tabla 3 se pueden observar los resultados de la misma para el año 2015, estos muestran cómo se va superando el nivel de estudiantes entre los puestos 1 al 400, donde nueve de los departamentos ubica a más del $40 \%$ de su población estudiantil en estos lugares. Si se observa la tabla 4, relación de la población estudiantil matriculada en el país, se estima que más de un millón de estudiantes buscan puntajes en las pruebas Saber $11^{\circ}$ con los niveles que les faciliten apoyo para acceder a la educación superior. El gran interrogante es si la oferta de la educación superior en el país tiene la capacidad de albergar esta población con una oferta de calidad.

Tabla 3

Informe del Ministerio de Educación y el ICFES. Pruebas Saber $11^{\circ}$ en 2015

\begin{tabular}{lclc}
\hline $\begin{array}{c}\text { ETC- Entidad Territorial } \\
\text { Departamental }\end{array}$ & $\begin{array}{c}2015, \\
\text { porcentaje \% }\end{array}$ & $\begin{array}{c}\text { ETC- Entidad Territorial } \\
\text { Departamental }\end{array}$ & $\begin{array}{c}2015, \\
\text { porcentaje \% }\end{array}$ \\
\hline Santander & 49,38 & San Andrés & 35,08 \\
\hline Cundinamarca & 49,12 & Vichada & 30,34 \\
\hline Arauca & 46,41 & Caquetá & 29,88 \\
\hline Norte de Santander & 44,40 & Tolima & 29,30 \\
\hline Casanare & 43,37 & Guaviare & 27,84 \\
\hline Boyacá & 43,16 & Cesar & 26,61 \\
\hline Nariño & 41,57 & Sucre & 25,19 \\
\hline Huila & 41,20 & Córdoba & 24,33 \\
\hline Guainía & 40,96 & Atlántico & 23,77 \\
\hline Meta & 40,69 & Vaupés & 23,64 \\
\hline
\end{tabular}




\begin{tabular}{lclc}
\hline $\begin{array}{l}\text { ETC- Entidad Territorial } \\
\text { Departamental }\end{array}$ & $\begin{array}{c}2015, \\
\text { porcentaje } \%\end{array}$ & $\begin{array}{c}\text { ETC- Entidad Territorial } \\
\text { Departamental }\end{array}$ & $\begin{array}{c}2015, \\
\text { porcentaje } \%\end{array}$ \\
\hline Quindío & 40,63 & La Guajira & 22,17 \\
\hline Risaralda & 39,52 & Cauca & 20,64 \\
\hline Putumayo & 39,21 & Amazonas & 18,40 \\
\hline Caldas & 36,08 & Bolívar & 16,74 \\
\hline Valle del Cauca & 36,07 & Magdalena & 14,79 \\
\hline Antioquia & 36,00 & Chocó & 9,33 \\
\hline
\end{tabular}

Fuente: Ministerio de Educación de Colombia, 29 de octubre de 2015

\section{Conclusiones}

Para los estudiantes de primaria y secundaria en Colombia se preparan pruebas nacionales a los grados $3^{\circ}, 5^{\circ}, 9^{\circ}$ y $11^{\circ}$ y se participa en las distintas pruebas internacionales. Los resultados académicos en las pruebas son muy bajos y el país se ha debatido entre el dilema de la cobertura y la calidad y los esfuerzos por mejorar la desigualdad entre los niveles lo ha llevado a mejorar la cobertura, pero esto no se evidencia en los desempeños de los escolares.

Hay debilidad en acceso y progresión oportuna hacia y dentro de cada uno de los niveles del sistema educativo. Un 92\% de la población es atendida en la primaria, pero un $21 \%$ de los estudiantes presentan atraso en el sistema, lo que ocasiona bajos resultados en las evaluaciones. El sistema logra ubicar un $71 \%$ de los jóvenes en el nivel de secundaria, pero un escaso $40 \%$ acceden al nivel de secundaria superior llevando al país a estar ubicado entre los últimos seis países de la región que completan el ciclo de educación obligatoria de sus jóvenes.

En la búsqueda de resultados en pruebas nacionales e internacionales, el sector ha realizado múltiples intentos ajustados a distintas normas, pero sin transformar significativamente los resultados. Los esfuerzos se concentran en adiestrar a los estudiantes en la tipología de las evaluaciones, en los contenidos de las asignaturas o áreas y en el manejo de tipos de textos.

Se hace un manejo fragmentado de la información con poca presencia de propuestas que transversalicen e integren la información, lo que dificulta al estudiante dar respuesta a posibles alternativas de solución que se le plantean en las evaluaciones. 
Se observa problemas de adecuación de los espacios, ambientes escolares, formación de maestros, número de estudiantes por capacidad instalada en aulas, así como una reciente insistencia de que lo evaluado en pruebas externas nacional o internacional debe ser lo atendido de manera exclusiva en el aula.

El nivel de cada una de las competencias evaluadas es aún preocupante. Los resultados de las pruebas PISA para los años 2006 y 2009 registran aumentos en las tres áreas (matemáticas, lectura y ciencias). No obstante, el país se ubica en los rangos inferiores entre los países participantes. Para 11 grado el $44 \%$ de los estudiantes evaluados en pruebas nacionales en 2013 se ubicaban en el nivel bajo de la competencia matemática y en la prueba internacional PISA Colombia ocupaba el puesto 62 en este componente de 65 países presentados.

Para el periodo 2012 a 2015 más del 50\% de los estudiantes de 5 grado se acumulan en los niveles de desempeño mínimo e insuficiente de las áreas de lenguaje y matemáticas. En 9 grado el porcentaje aumenta a más del $60 \%$ en matemáticas con una tendencia al 48 y $50 \%$ en lenguaje.

Los resultados en las instituciones oficiales se registran por debajo de las instituciones privadas del país. Se puede observar en los resultados de las pruebas internacionales de lenguaje, matemáticas y ciencias aplicadas en los años 2006, 2009 y 2012.

Para 2015 se observa que se va superando el nivel de estudiantes entre los puestos 1 al 400, donde nueve de los departamentos ubica a más del $40 \%$ de su población estudiantil en estos lugares. La oportunidad de becas para el acceso a la educación superior ha motivado a más de un millón de estudiantes a buscar puntajes en las pruebas Saber $11^{\circ} \mathrm{con}$ los niveles que les permitan acceder a estos apoyos convirtiéndose en un estímulo para mejorar resultados.

Según la OECD (2019) el rendimiento de Colombia en lectura en PISA 2018 fue menor que el registrado en 2015, si se considera un periodo más largo, el desempeño medio mejoró en todas las materias -incluso la lectura- desde que el país participó por primera vez en PISA en 2006.

Las debilidades del sistema educativo tienen dos problemas por atender y debe diferenciarse las estrategias que buscan mejorar la cobertura de aquellas que deben fortalecer el trabajo en el aula para elevar los resultados en los desempeños de los escolares al presentar las pruebas nacionales y las internacionales tanto en la básica como en la media. 
Si se tiene en cuenta que en Colombia la educación se define como un proceso de formación permanente, personal cultural, social y fundamentado en la concepción integral de la persona, su dignidad, derechos y deberes. Entonces se debe fortalecer el programa que incremente la calidad a partir de procesos de autoevaluación de las instituciones educativas y las secretarías de educación para elaborar planes de mejoramientos pertinentes a las necesidades de cada contexto.

Una combinación de las dinámicas institucionales y de la comunidad tales como evaluaciones a las estrategias pedagógicas, a los procesos de enseñanza y aprendizaje, a la combinación de los insumos didácticos, tecnológicos y físicos a disposición de docentes y estudiantes; a la actualización y formación de los docentes y directivos, a la asignación de recursos financieros, a la participación de la comunidad educativa hace efectivo el logro de las metas educativas.

Se fortalece la propuesta del aula con un trabajo integral docente- estudiante-escuela- comunidad que a partir de un diagnóstico de las necesidades y sus alternativas de solución lleve al desarrollo de las habilidades y destreza necesarias para el desempeño de las competencias en la solución de situaciones cotidianas para obtener los resultados esperados.

El diálogo entre saberes, la participación en grupos de investigación, la consolidación de redes de trabajo académico, el juego de roles en la presentación de alternativas de solución a situaciones reales, el fortalecimiento de las disciplinas, los intercambios entre pares y el desarrollo de las competencias básicas y blandas son propuestas que dinamizan el trabajo pedagógico y animan a los actores en el desarrollo de las responsabilidades personales, académicas y el mejoramiento de resultados personales y sociales. Preparar para la vida es preparar para dar respuesta correcta a situaciones planteadas en las pruebas, por ende, los resultados de las evaluaciones cambian.

\section{Referencias}

Arancibia, V. (1997). Los sistemas de medición y evaluación de la calidad de la educacion. Chile: UNESCO. Recuperado de https://unesdoc.unesco.org/ark:/48223/ pf0000183651

Ayala-García, J. (2015). Evaluación externa y calidad de la educación en Colombia. Documento de trabajo sobre economía regional 217. Cartagena: Banco de la República. Centro de Estudios Económicos Regionales (CEER). 
Pruebas de evaluación Saber y PISA en la Educación Obligatoria de Colombia luz Amparo Sanabria James, M. Carmen Pérez Almagro y luis Enrique Riascos Hinestroza

Banco de la República. (2015). Evaluación externa y calidad de la educación en Colombia. Documentos de trabajo sobre economía regional, 45.

Castells, M. (2000). Globalización, Estado y sociedad civil: el nuevo contexto histórico de los derechos humanos. ISEGORIA 22, 5-17.

Centro Virtual de Noticias de Educación. (28 de noviembre de 2014). Así están las regiones del país según resultados de las Pruebas Saber $11^{\circ}$. CVNe, Centro Virtual de Noticias de Educación, p. 1.

Centro Virtual de Noticias en Educación. (28 de octubre de 2015). Colombianos: conozcan los resultados de las Pruebas Saber $11^{\circ}$ en las regiones del país. CVNe. Centro Virtual de Noticias en Educación, p. 1.

Decreto 088 de 1976, por el cual se reestructura el sistema educativo y se reorganiza el Ministerio de Educación Nacional. Recuperado de https://www.acnur.org/fileadmin/ Documentos/BDL/2008/6506.pdf

Decreto 230 de 11 de febrero de 2002, por el cual se dictan normas en materia de currículo, evaluación y promoción de los educandos y evaluación institucional.

Decreto 1002 de 24 de abril de 1984, por el cual se establece el Plan de Estudios para la Educación Preescolar, Básica (primaria y secundaria) y Media Vocacional de la Educación Formal Colombiana.

Decreto 1290 de 16 de abril de 2009, por el cual se reglamenta la evaluación del aprendizaje y promoción de los estudiantes de los niveles de educación básica y media.

Decreto 1419 de 17 de julio de 1978, por el cual se señalan las normas y orientaciones básicas para la administración curricular en los niveles de educación preescolar básica (primaria y secundaria) media vocacional e intermedia profesional.

Decreto de 1469 de 3 de agosto de 1987, por el cual se reglamenta el artículo $8^{\circ}$ del Decreto Ley 088 de 1976 sobre promoción automática para el nivel de educación básica primaria.

Decreto 1860 de 3 de agosto de 1994, por el cual se reglamenta la Ley 115 de 1994, en los aspectos pedagógicos y organizativos generales.

Delgado, M. (2014). La Educación Básica y Media en Colombia: Retos en Equidad y Calidad. Bogotá: Fedesarrollo. Centro de Investigación Económica y social. Recuperado de https://www.repository.fedesarrollo.org.co/bitstream/handle/11445/190/La\%20educaci\%C3\%B3n\%20b\%C3\%A1 sica\%20y\%20media\%20 en $\% 20$ Colombia $\% 2$ retos $\% 20$ en $\% 2$ equidad $\% 20 y \% 20$ calidad $\% 20-\% 20$ KAS. pdf? sequence $=2$ \&isAllowed $=y$

Grupo de Investigación sobre Pruebas Masivas en Colombia. (2008). El surgimiento del Servicio Nacional de Pruebas del ICFES en las voces de sus protagonistas 2(3), pp. 115-134.

Horn, R., Wolff, L., \& Vélez, E. (1992). Sistemas de evaluación educacional en América Latina. Reseña temática y experiencias recientes. Proyecto principal de educación en América Latina y el Caribe. Boletín 27. Santiago de Chile: UNESCO, Oficina Regional de Educación para América Látina (OREALC). Recuperado de https://unesdoc.unesco. org/ark:/48223/pf0000091968_spa

Hurtado de Barrera, J. (2000). Metodología de la investigación holística. Caracas: Fundación Sypal ( $3^{\mathrm{a}} \mathrm{Ed}$.). 
Pruebas de evaluación Saber y PISA en la Educación Obligatoria de Colombia

ICFES (abril de 2018). Informe de resultados nacionales saber $3^{\circ}, 5^{\circ}$ y $9^{\circ} 2012-2017$. Bogotá, Colombia. www.icfes.gov.co

Instituto Colombiano para la Evaluación de la Educación ICFES. (2019). Recuperado de https://www.icfes.gov.co/resultados-saber-pro

Instituto Colombiano para la Evaluación de la Educación ICFES. (2019). Recuperado de https://www.icfes.gov.co/

Las distintas Pruebas. (enero-marzo de 2006). Al Tablero 38. El periódico de un país que educa y se educa. Recuperado de https://www.mineducacion.gov.co/1621/propertyvalue-33074.html

Ley 115 de febrero 8 de 1994, por la cual se expide la ley general de educación. Recuperado de https://www.mineducacion.gov.co/1621/articles-85906_archivo_pdf.pdf

Los Tres Editores SAS. (28 de octubre de 2015). Colombianos: conozcan los resultados de las Pruebas Saber $11^{\circ}$ en las regiones del país. Recuperado de http://www.lostreseditores.com/colombianos-conozcan-los-resultados-de-las-pruebas-saber-11-enlas-regiones-del-pais/

Ministerio de Educación Nacional (MEN) (2020). https://www.mineducacion.gov.co/portal/Normatividad

Mora, A. I. (2004). La evaluación educativa: conceptos, períodos y modelos. Actualidades Investigativa en Educación., 4(2), 1-28. Recuperado de https://www.redalyc.org/ pdf/447/44740211.pdf

OECD. (2019). PISA 2018 Results (Volume III): what school life means for students' lives. https://doi.org/10.1787/acd78851-en

OEl, Conferencia Iberoamericana de Ministros de Educación. (julio de 2009). Facebook, metas educativas 2021. Recuperado de http://www.oei.es/metas2021/foroevaluacion.htm

Pérez, R. (2000). La evaluación de programas educativos: conceptos básicos, planteamientos generales, políticas. Revista de Investigación Educativa 18 (2), 261-287.

Reimers, F. (2003). El contexto social de la evaluación educativa en América Latina. Revista Latinoamericana de Estudios Educativos XXXIII(003), 9-52.

Rodríguez, L. H. (2 de marzo de 2018). Las pruebas externas, una opción para mejorar los puntajes. El Espectador. Recuperado de https://www.elespectador.com/noticias/educacion/las-pruebas-externas-una-opcion-para-mejorar-los-puntajes-articulo-740947

Pruebas Pisa: Colombia uno de los pocos que mejora pero sigue por debajo de la media. (12 de junio de 2016). Semana. Recuperado de https://www.semana.com/on-line/ articulo/resultados-colombia-pruebas-pisa-2015/508330

Peppler-Barry, U. \& Fiske, E. B. (2000). Foro Mundial sobre la Educación, Dakar, Senegal, 26-28 de abril de 2000: informe final. Francia: UNESCO. Recuperado de https:// unesdoc.unesco.org/ark:/48223/pf0000121117_spa

VV.AA. (2008). Reflexiones en torno a la evaluación de la calidad educativa en América Latina y el Caribe. Santiago de Chile: Publicado por la Oficina Regional de Educación de la UNESCO para América Latina y el Caribe (OREALC/UNESCO Santiago) y el Laboratorio.

VV.AA. (2010). Metas Educativas 2021. La educación que queremos para la generación de los bicentenarios. Madrid: Organización de Estados Iberoamericanos para la Educación, la Ciencia y la Cultura (OEI) 